\title{
High-accuracy deep-UV Ramsey-comb spectroscopy in krypton
}

\author{
Sandrine Galtier ${ }^{1} \cdot$ Robert K. Altmann $^{1} \cdot$ Laura S. Dreissen $^{1} \cdot$ Kjeld S. E. Eikema $^{1}$
}

Received: 1 September 2016 / Accepted: 7 November 2016 / Published online: 20 December 2016

(c) The Author(s) 2016. This article is published with open access at Springerlink.com

\begin{abstract}
In this paper, we present a detailed account of the first precision Ramsey-comb spectroscopy in the deep UV. We excite krypton in an atomic beam using pairs of frequency-comb laser pulses that have been amplified to the millijoule level and upconverted through frequency doubling in BBO crystals. The resulting phase-coherent deep-UV pulses at $212.55 \mathrm{~nm}$ are used in the Ramseycomb method to excite the two-photon $4 p^{6} \rightarrow 4 p^{5} 5 p[1 / 2]_{0}$ transition. For the ${ }^{84} \mathrm{Kr}$ isotope, we find a transition frequency of 2829833101679(103) kHz. The fractional accuracy of $3.7 \times 10^{-11}$ is 34 times better than previous measurements, and also the isotope shifts are measured with improved accuracy. This demonstration shows the potential of Ramsey-comb excitation for precision spectroscopy at short wavelengths.
\end{abstract}

\section{Introduction}

Quantum electrodynamics (QED) theory is a cornerstone of the standard model and one of the best tested fundamental theories in physics. Its predictions have been verified with extreme precision, e.g., by measuring the fine structure constant $\alpha$ derived from measurements of the electron $g$-factor $[1,2]$, interferometric recoil experiments [3, 4],

This article is part of the topical collection "Enlightening the World with the Laser" - Honoring T. W. Hänsch guest edited by Tilman Esslinger, Nathalie Picqué, and Thomas Udem.

Robert K. Altmann

rkaltmann@gmail.com

1 LaserLaB, Department of Physics and Astronomy, VU University Amsterdam, De Boelelaan 1081, 1081 HV Amsterdam, The Netherlands and bound-state QED tests based on precision spectroscopy in atoms, molecules and highly charged ions (see, e.g., [510]). Moreover, in the pursuit of testing QED ever better, substantial efforts have been made to extract fundamental quantities such as the Rydberg constant $R_{\infty}$ and the proton charge radius. Both can be obtained from spectroscopy of atomic hydrogen, assuming that QED is sufficiently precise. However, when the CREMA collaboration determined the proton charge radius from spectroscopy in muonic hydrogen (consisting of a proton and a muon), it leads to a considerable $(7 \sigma)$ mismatch with the value extracted from normal (electronic) hydrogen [11-13]. This mismatch, known as the proton radius puzzle, remains to be explained and requires more spectroscopic measurements, e.g., in systems other than (muonic) hydrogen. Recent results on muonic deuterium [14] reveal that also the deuteron radius is significantly smaller $(7.5 \sigma)$ than the radius based on normal deuterium spectroscopy.

Interesting candidates for precision spectroscopy to solve this puzzle need to be sufficiently simple for precise theoretical treatment. One example is molecular hydrogen, made possible by recent improvements in theory [15]. Another is $\mathrm{He}^{+}$[16], which can be compared to muonic- $\mathrm{He}^{+}$spectroscopy [13]. The experimental challenge is the short wavelengths required for excitation, which ranges from the deep UV $(\approx 200 \mathrm{~nm})$ for $\mathrm{H}_{2}$ to extreme ultraviolet (XUV, $\lambda<60 \mathrm{~nm}$ ) for $\mathrm{He}^{+}$.

Such short wavelengths are typically obtained by frequency upconversion of near-infrared lasers in nonlinear crystals or noble gases. One can use a frequency-comb (FC) laser as the fundamental laser and take advantage of its excellent spectral resolution and pulse peak power, to perform direct frequency-comb spectroscopy (DFCS) [17-19]. To achieve sufficient upconversion to the UV or XUV range, several approaches have been investigated. 
This includes the use of enhancement resonators with intracavity high harmonic generation [19, 20], and amplification at full repetition rate $[21,22]$, typically leading to infrared pulse energies in the $\mu \mathrm{J}$ range.

We developed a different approach, based on amplification of only two FC pulses, which combines precision spectroscopy with high-power laser pulses. Amplification of only two pulses leads to orders of magnitude higher pulse energy in the $\mathrm{mJ}$ range. The accuracy and resolution are recovered in this case by recording a series of Ramseytype signals, leading to Ramsey-comb spectroscopy (RCS). It features a strong suppression of systematic shifts such as the AC-Stark shift and chirp-induced effects, which are common problems in metrology experiments. The Ramsey-comb method has previously been demonstrated in the infrared on two-photon transitions in rubidium and cesium [23]. Recently we reported on the extension to the deepUV region [24], and here we present a detailed account of this experiment. We demonstrate its potential by exciting the $4 p^{6} \rightarrow 4 p^{5} 5 p[1 / 2]_{0}$ two-photon transition in ${ }^{84} \mathrm{Kr}$ at $212.55 \mathrm{~nm}$, and we also report improved isotope shift measurements for the other isotopes.

\section{Ramsey-comb spectroscopy principle}

We recall here the key features of Ramsey-comb spectroscopy; a more detailed description can be found in [23, 25]. As the name indicates, the method combines Ramsey spectroscopy [26] with frequency-comb (FC) lasers [27, 28]. Excitation of an atom or molecule is performed with two (short) coherent laser pulses that are selected from a frequency-comb laser. The pulses can be amplified and therefore easily frequency upconverted to cover a wide spectral range (see Sect. 4). By taking the original pulses from a FC, their coherence can be maintained over long intervals (even seconds), and it becomes possible to record a series of Ramsey signals using different pulse pairs with a delay that is a multiple of the FC pulse repetition time. This we call Ramsey-comb spectroscopy (RCS), leading to interesting new properties compared to standard Ramsey spectroscopy.

It starts with excitation using two coherent pulses, leading to interference between the coherent superpositions induced by each pulse. If the time delay $\Delta T$ is changed between the two resonant pulses, while keeping the relative optical phase $\Delta \phi$ constant, we observe an oscillation of the population $\left|\rho_{\mathrm{e}}\right|^{2}$ of the excited state according to:

$\left|\rho_{\mathrm{e}}\right|^{2} \propto \cos \left(2 \pi f_{\mathrm{tr}} \times \Delta T+\Delta \phi\right)$

This Ramsey signal from two separate excitations is widely used in, e.g., atomic clocks [29]. To extract the atomic frequency $f_{\text {tr }}$, one must exactly know the time delay $\Delta T$ and the relative phase $\Delta \phi$ between the two pulses. These conditions are conveniently met by taking pulses from a frequency-comb laser. A frequency comb is a mode-locked laser whose spectrum comprises equally spaced optical frequencies (modes) separated by the repetition frequency

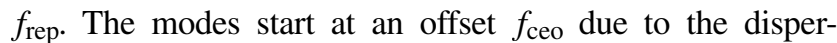
sion and nonlinear effects in the cavity, leading to a spectrum of $f_{n}=f_{\text {ceo }}+n f_{\text {rep }}$, where $n$ is an integer. In the time domain, the modes interfere to pulses with a time interval of $T_{\text {rep }}=1 / f_{\text {rep }}$, and with a carrier-envelope phase shift from pulse to pulse equal to $\Delta \phi_{\text {ceo }}=2 \pi f_{\text {ceo }} / f_{\text {rep. }}$. Both $f_{\text {ceo }}$ and $f_{\text {rep }}$ of the laser can be actively stabilized to an ultra-stable frequency reference, e.g., a cesium clock, leading to highly reproducible phase-coherent pulses, as required for Ramsey spectroscopy.

For the Ramsey-comb method, we select two pulses from the FC at multiples $N$ of the repetition time $T$, initially

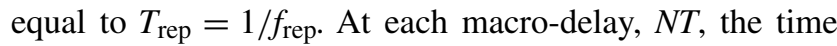
delay can be adjusted on a (much) smaller scale in steps of tens of attoseconds by tuning the repetition time $T_{\text {rep }}$ of the laser. Therefore, the time delay $\Delta T$ between the two pulses can be written as:

$\Delta T=N T+\delta t$

The micro-delay $\delta t$ scan provides the right time scale to probe the Ramsey oscillations of the atomic transition frequency. The ability to measure and combine Ramsey fringes from multiples of $N T$ permits a much more precise frequency determination because the Ramsey fringes can be probed on much longer timescales. In addition, the frequency is determined from the phases of the time domain Ramsey signals at each NT. Any phase shift of the Ramsey signals that is constant for each $N T$ will simply shift all the Ramsey fringes together, but will not affect the extracted the frequency. Therefore, any common phase shift does not influence the frequency determination which also enhances the accuracy (see [25]). This includes an optical phase shift between the excitation pulses. This is initially equal to $N \times \Delta \Phi_{\text {ceo }}$ from the FC, but is perturbed during the subsequent amplification process. The laser system is constructed such that this phase perturbation is constant (typically on the mrad level) as function of the macro-delay $N T$. Moreover, this also includes any constant phase shift of the atomic phase. For example, the AC-Stark shift manifests itself as a phase shift imprinted on the state superposition during the excitation. As long as the excitation pulse energy is kept constant for different macro-delays, the ACStark effect results in a constant phase shift of the Ramsey signals, which is therefore eliminated in the analysis procedure. The light-shift insensitive nature of the Ramsey-comb spectroscopy combined with high pulse energy for easy frequency conversion are two key advantages compared to conventional direct frequency-comb spectroscopy. 


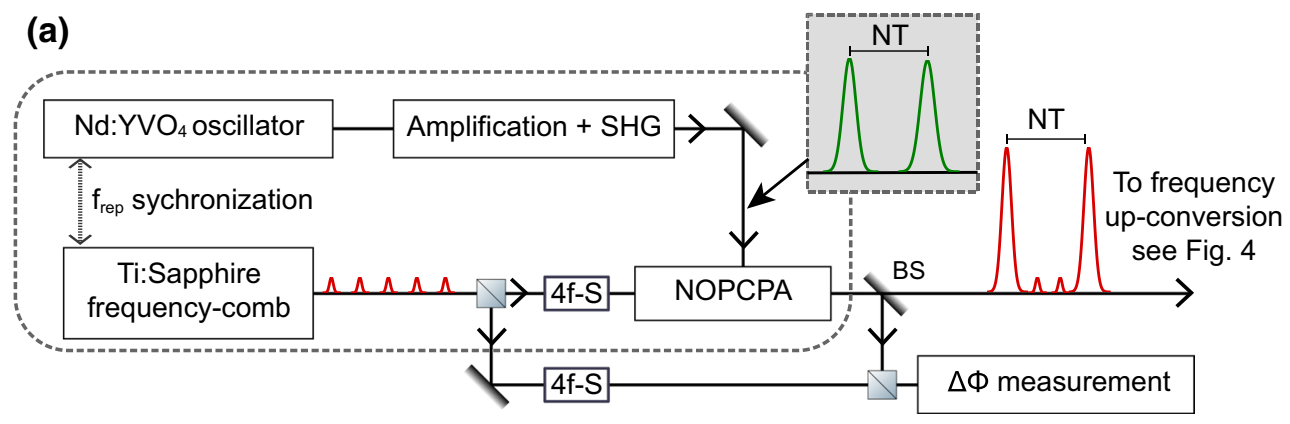

Fig. 1 Ramsey-comb laser system. Details of part $(a)$ about on the generation of the pump pulses and on the noncollinear optical parametric chirped-pulse amplification (NOPCPA) are given in Fig. 2. 4f-S 4f grating stretcher, $B S$ beamsplitter

\section{Laser system}

The experimental setup is shown in Fig. 1. The pulse pairs are extracted from a home-built mode-locked Ti:sapphire frequency comb, delivering $4 \mathrm{~nJ}$ femtosecond pulses at a repetition rate of $126.6 \mathrm{MHz}$. The frequency comb is fully referenced to an atomic Cs clock. A pulse pair is selectively amplified in a noncollinear optical parametric chirped-pulse amplifier (NOPCPA). The NOPCPA is designed to provide high gain over a broad spectrum (hundreds of nanometers) and consists of three passes in two BBO crystals [30, 31] (see Fig. 2). The system bandwidth is convenient for easy tunability, as the actual amplified spectrum of the comb laser for this experiment is only a few $n m$ wide.

The pump laser is detailed in Fig. 2. The starting point is a SESAM mode-locked $\mathrm{Nd}: \mathrm{YVO}_{4}$ laser producing 10 ps pulses. The pulse length is increased to 75 picoseconds by spectral clipping; two of them are then selected using fast modulators. Both pulses are then amplified to the 1-mJ level in a grazing-incidence side-pumped $\mathrm{Nd}: \mathrm{YVO}_{4}$ bounce amplifier (detailed in [32, 33]).

In the post-amplifier, the pulse pair is further amplified to $27 \mathrm{~mJ}$ per pulse, using a quasi-CW diode pumped $\mathrm{Nd}$ :YAG module $(6.35 \mathrm{~mm}$ rod diameter, type number REA6308-3P200H from Northrop Grumman). To obtain a top-hat seed intensity profile for this amplification stage, the beam from the bounce amplifier is enlarged to $6 \mathrm{~mm}$, and a center part is selected with a $3.5 \mathrm{~mm}$ diameter iris. To prevent diffraction hot spots, the uniform intensity profile at the iris position is relay imaged to the Nd:YAG rod and throughout the whole amplifier. The amplifier is set up in a two-pass configuration as illustrated in Fig. 2. A Faraday rotator is placed in between the two passes to compensate for birefringence and to couple out the back-reflected beam with a thin-film polarizer. The amplified beam is further relay imaged and doubled to $532 \mathrm{~nm}$ to be used as the pump beam for the parametric amplifier.
At each amplification stage of the pump laser, the second pulse is less efficiently amplified compared to the first one due to energy depletion. There is also gain loss from unwanted pulses that are not fully suppressed by the modulators that select the pulses at the start of the amplifier chain. To ensure a pump pulse pair with equal intensities in the NOPCPA, the amplitude of the first pulse is injected with less energy compared to the second one in the bounce amplifier using the modulators. In between the bounce amplifier and post-amplifier, the energy ratio is adjusted (cut) again by changing the timing of the rising slope of a Pockels cell. With active feedback on the Pockels cell timing, the intensity ratio can be controlled and kept constant.Before seeding the NOPCPA, the Ti:sapphire laser pulses are stretched to $12 \mathrm{ps}$ by adding a chirp of $1.2 \times 10^{6}$ $\mathrm{fs}^{2}$ combined with wavelength selection of the spectrum via a movable slit in the Fourier plane of a $4 \mathrm{f}$ grating stretcher. The sharp spectral clipping of the seeding beam results in a sinc-like pulse in the time domain, offering spectral power at its wings. These wings are strongly amplified in the NOPCPA operating in a saturated regime. Because we use chirped pulses, the amplification in the wings of the pulses corresponds to an enhanced amplification of the 'red' front edge and the 'blue' trailing edge of the pulse, resulting in a spectrum with two peaks as shown in Fig. 3. It also leads to a separation in time of the two spectral regions and increases the total amplified pulse duration to approximately $20 \mathrm{ps}$.

Only the FC pulses that overlap in time with the pair of pump pulses are amplified in the NOPCPA. Therefore, the choice of the pump pulse pair determines the time interval between the pair of amplified FC pulses. The FC pulses reach an energy of $1.5 \mathrm{~mJ}$ each, with a spectral bandwidth of approximately $3.5 \mathrm{~nm}$ centered around $850.2 \mathrm{~nm}$.

The parametric amplification process and self- and cross-phase modulation between all interacting beams can imprint a phase shift on the amplified pulses, depending mostly on the pump intensity $[31,34,35]$. Any differential 


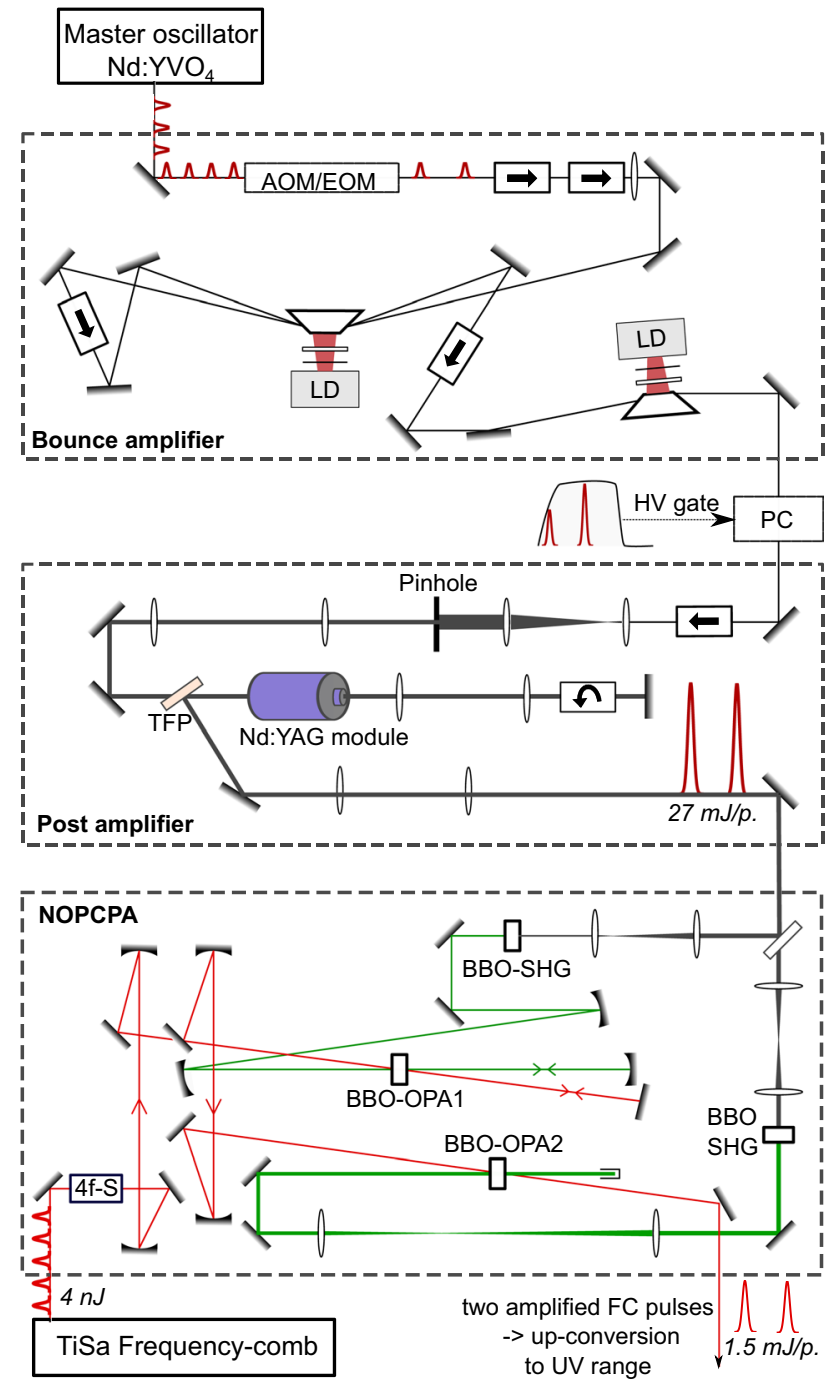

Fig. 2 Pump laser and parametric amplification stages. Two pulses from a master oscillator $\left(\mathrm{Nd}: \mathrm{YVO}_{4}\right)$ are selected and amplified in a double-pass bounce amplifier followed by a single-pass bounce amplifier. The post-amplifier stage consists of two passes in a Nd:YAG module gain medium producing pulses of $27 \mathrm{~mJ}$ each, which are used as the pump laser of the NOPCPA. The amplitude of the first pump pulse is adjusted via by the rising-slope timing of a PC gate, such as the two frequency-comb pulses have equal intensities. $P C$ Pockels cell, $L D$ laser diode, $T F P$ thin-film polarizer, $4 f-S$ ff grating stretcher

phase shift $\Delta \Phi$ between different pulse pairs can lead to a frequency shift of the excited two-photon transition according to:

$\Delta f=\frac{8 \times \Delta \Phi}{2 \pi \times N T}$

The factor 8 accounts for the two doubling stages to the UV $(\times 4)$ and the use of two photons to drive the atomic transition $(\times 2)$. To measure the influence of the amplification process on the phase of the FC pulses, we use a setup

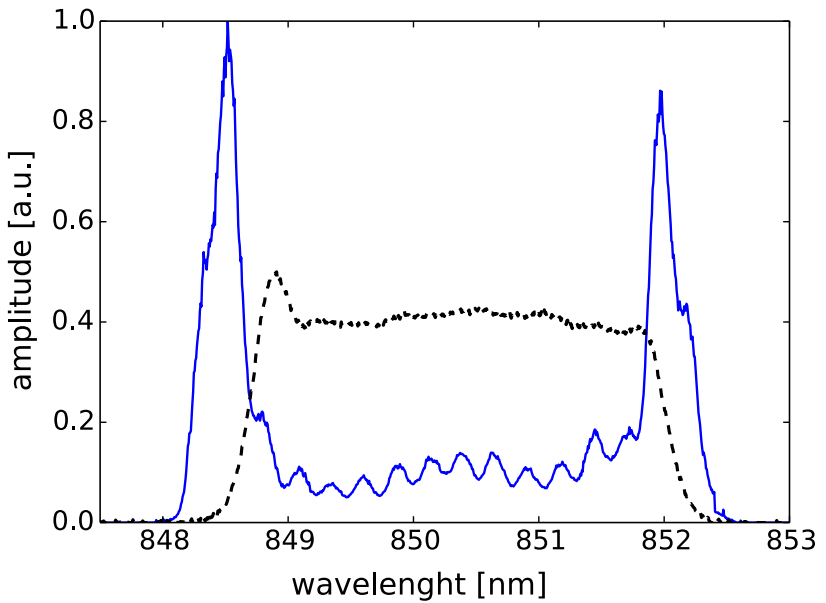

Fig. 3 Spectrum of the selected FC spectrum before amplification (in dash black line) and after amplification (solid blue line) in the NOPCPA

consisting of a Mach-Zehnder interferometer in combination with spectral interferometry $[31,35]$. The two amplified pulses are combined with their respective non-amplified FC pulses which are extracted before the amplification process using a combination of a wave plate and polarizing cube (see Fig. 1). The spectral interference between the amplified and original FC pulses is recorded with a camera. From these interference patterns, the relative phase induced by the amplification can be determined.

\section{Excitation scheme}

We excite the $4 p^{6} \rightarrow 4 p^{5} 5 p[1 / 2]_{0}$ two-photon transition in $\mathrm{Kr}$ using $50 \mu \mathrm{J}$ deep-UV pulses at $212.5 \mathrm{~nm}$. Two-photon spectroscopy allows the use of multiple pairs of comb modes leading to the same total energy over many nanometers of bandwidth leading to efficient use of power and increased signal to noise compared to one-photon transitions. The deep-UV pulses are generated from the amplified FC laser after successive doubling stages using thin $\mathrm{BBO}$ crystals (beta barium borate $-\beta-\mathrm{BaB}_{2} \mathrm{O}_{4}$ ). The first doubling stage is performed in a 1-mm-thick BBO crystal. At this stage, the entire spectrum of the fundamental light is doubled with an efficiency of approximately $35 \%$. We perform the second doubling with two different BBO crystals of $0.5 \mathrm{~mm}$ thickness, and a phase-matching bandwidth lower than $0.5 \mathrm{~nm}$. The phase-matching angle of the two last crystals can be adjusted such that they double independently the 'red' or 'blue' edge of the spectrum (see Fig. 4). Because of the cathedral-like shape of the input spectrum, this configuration still results in sufficient intensity in the ultraviolet. The doubling in the last stage is adjusted such that at the center wavelength of the atomic transition no 


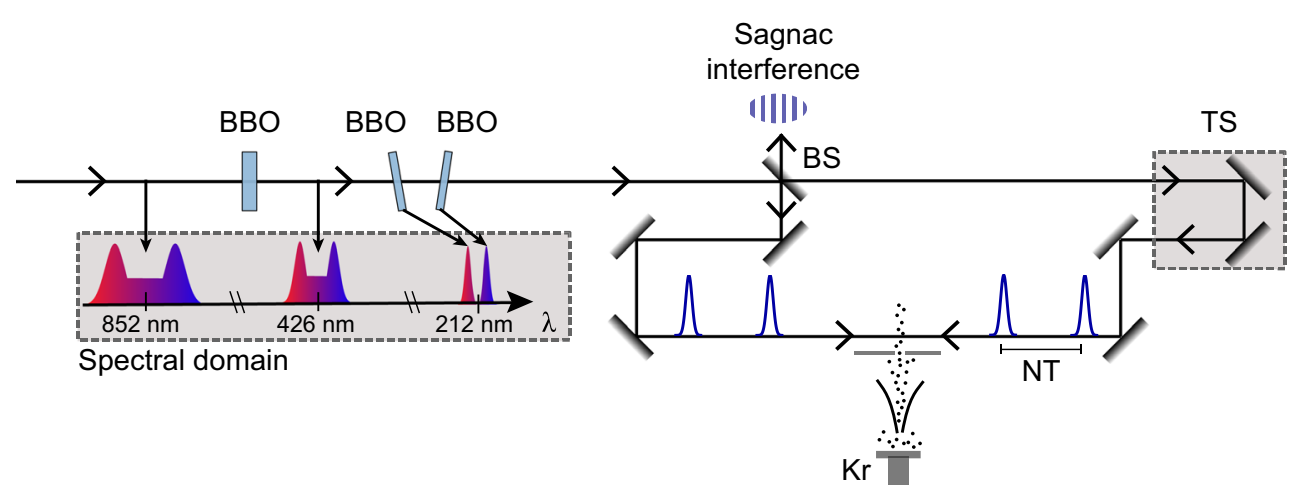

Fig. 4 Fourth harmonic of the amplified FC pulses is obtained using three different $\mathrm{BBO}$ crystals. The first crystal doubles the entire spectrum, the second and the third crystal double, respectively, the 'blue' part and the 'red' part of the spectrum (see inset spectral domain). The krypton atoms are excited in a counter-propagating configura-

light is produced. Therefore, excitation is only possible if the 'red' front edge and the 'blue' trailing edge of the pulses overlap in time to match the transition frequency. This is fulfilled by a combination of 'red' and 'blue' parts of the spectrum from pulses that travel from opposite sides. It leads to two spatial positions (where 'red' meets 'blue,' and 'blue' meets 'red') where excitation takes place with a suppressed first-order Doppler shift. At the same time, it eliminates the background signal one would otherwise get due to (Doppler broadened) two-photon excitation from one side.

The krypton atoms perpendicularly cross the two counter-propagating UV beams to minimize residual first-order Doppler effects. The atoms are produced in a highly collimated beam with a the combination of a pulsed valve and a double skimmer arrangement (with 0.5 and $3 \mathrm{~mm}$ wide aperture at 3 , and $25 \mathrm{~cm}$ distance to the nozzle, respectively). Because of the chirp in the pulses, the 'red' and 'blue' part of the spectrum are also spatially separated by about $6 \mathrm{~mm}$. This is bigger than the $3 \mathrm{~mm}$ diameter atomic beam, and therefore we are able to observe the two collision points where 'red' meets 'blue,' and 'blue' meets 'red' separately (by using a delay line in one of the UV paths to position one of them into the atomic beam). Measurements for both collision points (which were in agreement with each other) have been combined for the final result. After the two-photon excitation, the atoms are state selectively ionized with a $532 \mathrm{~nm}$ pulse of a few mJ. It always arrives $4 \mathrm{~ns}$ after the second excitation pulse because it is derived from the second pump pulse of the NOPCPA. The generated ions are extracted by applying a $300 \mathrm{~V}$ electric potential. This extraction field operates in a pulsed mode to avoid any DC-Stark effect. Because of the bandwidth of the excitation pulses, all isotopes of krypton $\left({ }^{80} \mathrm{Kr},{ }^{82} \mathrm{Kr}\right.$, tion to reduce the first-order Doppler effect. After ionization with a $532 \mathrm{~nm}$ pulse (not shown), the ions are detected after a time-of-flight mass selector with a channel electron multiplier. $B S$ metallic beamsplitter, $T S$ translation stage

${ }^{83} \mathrm{Kr},{ }^{84} \mathrm{Kr}$, and ${ }^{86} \mathrm{Kr}$ ) are excited at the same time. Their respective ions are separated in time by a time-of-flight (ToF) detection scheme, after which they are individually detected with an extended dynamical range, channel electron multiplier (EDR-CEM) detector (SJUTS $\left.{ }^{\circledR}\right)$. We used the most abundant ${ }^{84} \mathrm{Kr}$ isotope for the determination of the absolute transition frequency and measured the isotopes relative to this.

\section{Experimental results and analysis}

One RCS measurement consists of the observation of two scans of the Ramsey oscillation at two different macrodelay between the deep-UV pulses. We start at $N=2$ to avoid any transient effects from the first pulse influencing the signal of the second pulse, e.g., in monitoring the pulse energy using the signal from a photodiode. We choose $N=7$ for the second scan as a compromise between a large time interval $\Delta T$ which is beneficial for the accuracy (as for any Ramsey-type experiment), and a good signalto-noise ratio (that deteriorates for longer delays due to the finite lifetime of the excited state of $27 \mathrm{~ns}$ ).

An individual scan is performed by varying the repetition time of the frequency comb over a range of $\delta t$ such that two periods of the Ramsey oscillations are recorded with a sampling of 14 points. Each point represents an average of the signal amplitude over 350 laser shots. We obtain a complete RCS measurement (two Ramsey oscillations at two different $N$ ) in approximately 6 minutes, as shown in Fig. 5. Such a recording is performed in a back-and-forth sequence, where even numbered data points at $N=2$ and $N=7$ are measured first followed by the odd-numbered data points at $N=7$ then $N=2$, to reduce the influence of drift effects (e.g., beam pointing). 

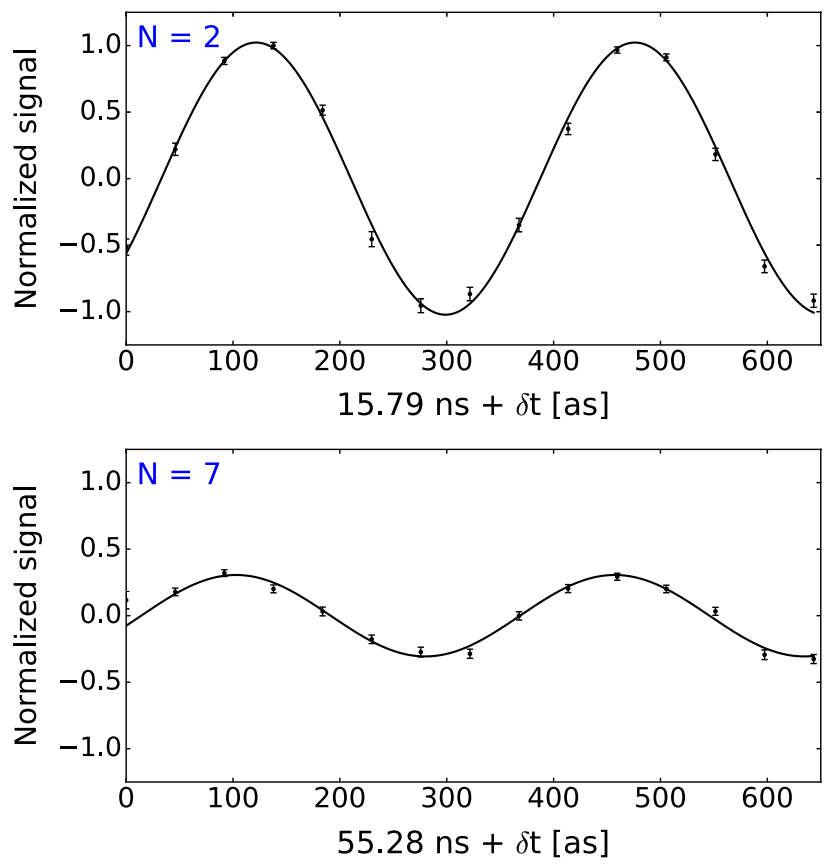

Fig. 5 Ramsey oscillations observed after the excitation of the atomic transition in ${ }^{84} \mathrm{Kr}$ by two different pairs of pulses. The two UV pulses are delayed by $2 \times T=15.79 \mathrm{~ns}$ in the upper graph and by $7 \times T=55.28 \mathrm{~ns}$ in the lower graph. The micro-delay $\delta t$ is scanned by adjusting the repetition rate of the frequency comb. The amplitude of the Ramsey signal decreases for longer delays because of the finite lifetime ( $\tau=27 \mathrm{~ns}$ ) of the excited state

At each $N$, a cosine is fitted to the Ramsey-fringe based on Eq. 1. The atomic frequency is then found based solely on the phases extracted from these fits [25]. In the simple case when only a single transition is excited, the phase evolves linearly with time, so that only two values of $N$ are needed to extract the atomic frequency. In the RCS method, the frequency is extracted modulo the frequency sampling interval (the biggest interval corresponds to a frequency spacing of $\left.f_{\text {rep }}\right)$. However, the previous determination [17] is precise enough to resolve the ambiguity. Otherwise, repeating the measurement at different repetition rate would straightforwardly solve it.

We will now discuss several potential sources of systematic errors.

\subsection{Amplification-induced phase shifts}

In order to determine that the amplification process did not alter the relative phase between the excitation-pulse pairs, the relative phase was measured with spectral interferometry (see Sect. 3). Even though absolute phase shifts between the amplified pulses can be on the order of 100 mrad (depending on, e.g., laser alignment), we measured no relative phase shift within a statistical uncertainty

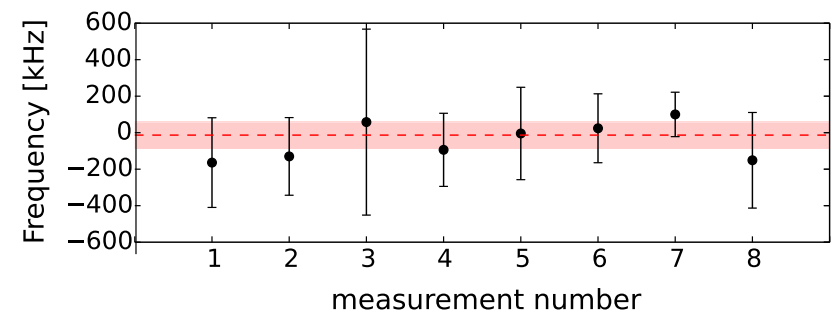

Fig. 6 Light-shift determination. Every point in this graph represents an AC-Stark shift determination (each based on typically 20 scans) by extrapolating the transition frequency to zero pulse energy from pairs of Ramsey-comb measurements with 25 and $50 \mu \mathrm{J}$ UV pulse energy. No frequency shift is observed within the statistical uncertainty of this measurement of $72 \mathrm{kHz}$

of $1 \mathrm{mrad}$ between $N=2$ and $N=7$. This translates into an uncertainty of $35 \mathrm{kHz}$ in the final determination of the atomic frequency.

\subsection{The AC- and DC-Stark shift}

The AC-Stark shift (or light shift) is a common major source of uncertainty for high-resolution spectroscopy using high laser intensities. The atomic frequency extracted by the RCS method is not affected by the ACStark shift as long as the induced phase-shift is the same for every Ramsey scan. This is ensured by keeping the amplitude of the infrared light-pulses stable within $1 \%$ using active feedback via the timing of the Pockels cell gate between the bounce and post-amplifier, as explained previously. To detect any possible residual AC-Stark effect in the UV, we performed a conventional test by varying the UV light pulse intensity with a factor two (using a polarizer and half-wave plate just before the doubling stages). This procedure also tests potential phase shifts induced in the doubling stages itself [17]. The result, illustrated in Fig. 6, indicates no AC-Stark shift or amplitude-related shift, within a uncertainty of $72 \mathrm{kHz}$. This uncertainty is comparatively large in view of the other sources of uncertainty due to a relatively low number of measurements that were taken to determine this effect.

To extract the ionized krypton atoms for detection, electric fields are applied that can potentially lead to a DCStark shift. To enable (nearly) field-free excitation, the extraction field was operated in a pulsed mode. For this mode, we calculated a residual electric field of at most 0.17 $\mathrm{V} / \mathrm{cm}$. No significant shift was seen when the transition frequency was also measured in a DC electric field of $29.4 \mathrm{~V} /$ $\mathrm{cm}$. Therefore, a negligible effect on the final transition frequency of $<1 \mathrm{kHz}$ is expected under the conditions of the pulsed extraction field. 


\subsection{Doppler shift}

The two-photon excitation is performed in a counter-propagating beam configuration in order to reduce first-order Doppler effects. To create the counter-propagating beams, the UV pulses are split using a metallic $50 \%$ beamsplitter (see Fig. 4). When the UV pulses combine again on the beamsplitter after one round trip through the vacuum apparatus, the exit port shows an interference pattern depending on the alignment of the UV beams. Perfectly counterpropagating beams cancel each other at the beamsplitter, leading to a single dark fringe. This procedure guarantees the collinearity of the two excitation beams with a precision better than $0.01 \mathrm{mrad}$ [36]. Such an angular mismatch can give rise to a frequency shift of $18 \mathrm{kHz}$ according to the following equation [36]:

$\Delta f=\theta_{1} \frac{\|v\|}{\lambda}$

where $\theta_{1} \ll 1$ is the angle between the two UV beams, $v$ is the atomic velocity and $\lambda$ is the excitation wavelength. Another source of first-order Doppler shift stems from our particular resonance condition which requires two excitation pulses with different frequencies (to cancel excitation from a single side). Therefore, even if the light beams perfectly overlap, they must also be exactly perpendicular to the atomic beam. An angular mismatch of $\theta_{2}$ between the light and the atomic beams leads to a frequency error:

$\Delta f=\Delta \nu_{\mathrm{UV}} \times \frac{\|v\| \cos \left(\pi / 2+\theta_{2}\right)}{c}$

where $c$ is the speed of light and $\Delta v_{\mathrm{UV}}$ is the frequency difference between 'blue' and the 'red' component of the spectrum. Moreover, the use of chirped pulses can lead to a systematic effect as explained in [6], named chirp-induced first-order Doppler shift (CIFODS). All these effects can be minimized by finding the angle $\theta_{2}$ for which the average first-order Doppler shift vanishes (when the UV beams are effectively perpendicular to the atomic beam).

To find this situation, we measure the atomic transition frequency for different velocities of the atomic beam. The velocity can be increased by using a mixture of krypton with neon (in a 1:5 ratio) or a mixture of krypton and helium (in a 1:9 ratio). The velocities of pure $\mathrm{Kr}$ and the mixtures have been measured using the approximately 40 $\mathrm{cm}$ long time-of-flight detection setup. After the deep-UV light excitation, the created ions are directed upward using an extraction field in the vertical direction. Two deflection plates, placed on both sides of the ions trajectory, steer the atomic beam on the detector. The velocity of the krypton atoms can then be calculated based on the geometry of the detection setup and the applied electric fields to steer the ions, see Fig. 7. We determined a velocity of the pure

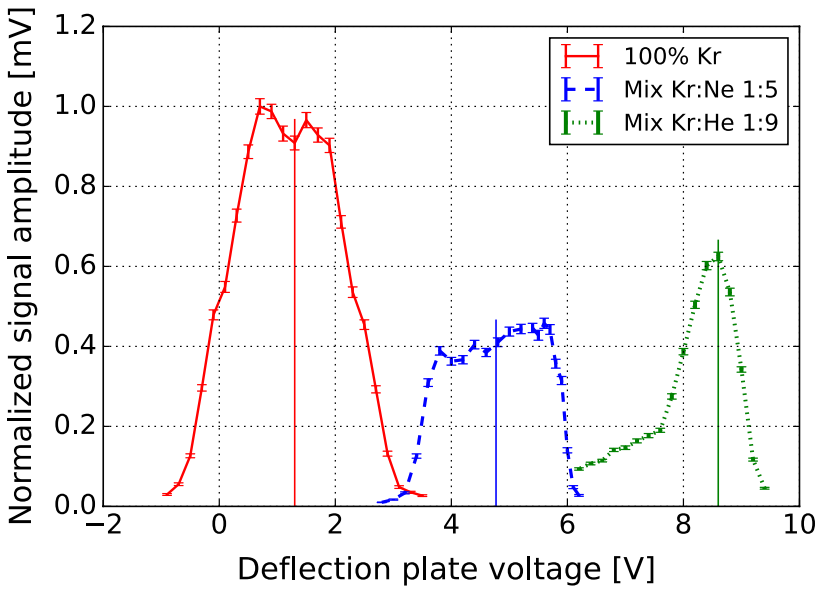

Fig. 7 Determination of the forward velocity of krypton atoms in the different gas mixtures, based on the voltage to deflect the ions in the ToF setup. The vertical lines show the voltage used to estimate the velocity of the two different gas mixtures. The signal amplitudes are normalized on the highest signal of pure $\mathrm{Kr}$

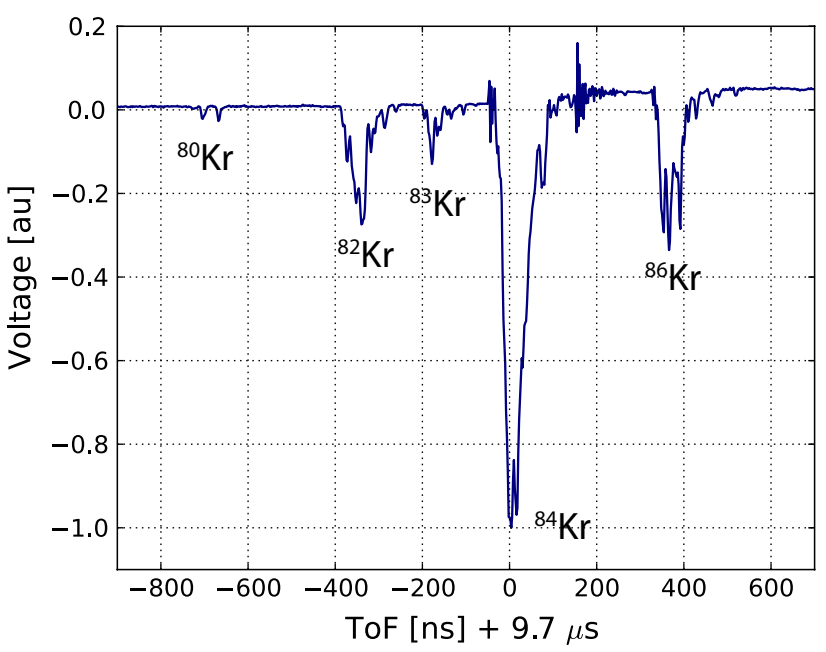

Fig. 8 Typical time-of-flight detection of the isotopes of krypton. The relative signal depends on the state of the phase of their respective Ramsey oscillation. We have chosen a time-of-flight snapshot which pattern is a close representation of their natural abundance. The large signal of the most abundant isotope ${ }^{84} \mathrm{Kr}$ strongly affects the operation of the detector, and therefore the signal of isotopes arriving later. This effect can also be seen as a shift of the detector signal baseline after ${ }^{84} \mathrm{Kr}$

krypton beam of $380(80)$, 686(60) $\mathrm{m} / \mathrm{s}$ for the kryptonneon mixture, and 931(134) $\mathrm{m} / \mathrm{s}$ for the krypton-helium mixture.

The angle between the UV beams and the atomic beam is tuned until the difference in transition frequency between the different gas mixtures is undetectable within the statistical uncertainty. The atomic frequency is then extracted by extrapolating to zero velocity based on pairs 

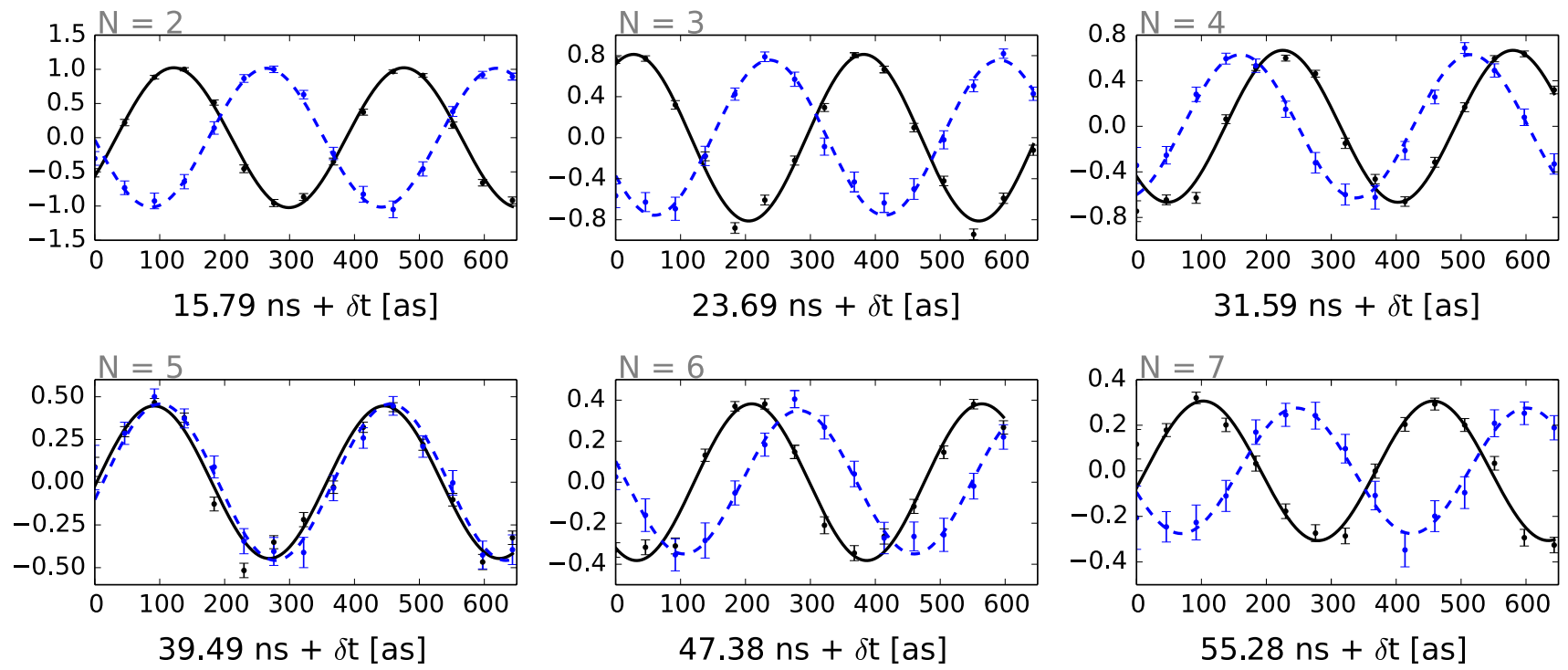

Fig. 9 Example of the isotope shift measurements. Multiple Ramsey scans for ${ }^{84} \mathrm{Kr}$ (solid black line) and ${ }^{82} \mathrm{Kr}$ (dashed blue line) for pulse pairs ranging from $N=2$ to $N=7$ are shown. For each $N$, the signals of the two isotopes have been acquired simultaneously. Because of the difference in transition frequency, the phases of the Ramsey scans evolve differently in time. The isotope shift can be determined from the relative phase difference at each value of $N$

spanning over roughly $800 \mathrm{~ns}$, as shown in Fig. 8. Each peak is equivalent to the detection of approximately $1-10$ ions within roughly $20 \mathrm{~ns}$. The EDR-CEM detector is not specified for such high count rates. As a result, the signal from isotopes arriving early in the ToF might influence the signal from isotopes arriving later. This effect potentially distorts the Ramsey signal of the heavier isotopes. The situation is even more subtle, because the amplitudes of the five isotopes signals evolve differently as function of time. To estimate the influence of the detector we performed the Ramsey-comb analysis procedure with different pulse delays (i.e., different $N$ ). Figure 9 shows the simultaneous acquisition of the Ramsey fringes for the isotopes ${ }^{82} \mathrm{Kr}$ and ${ }^{84} \mathrm{Kr}$ for $N=2$ to 7 . The isotope shift $f_{84 \mathrm{Kr}}-f_{82 \mathrm{Kr}}$ can be extracted from every single $N$ scan. No systematic dependence on $N$ has been observed for the isotopes ${ }^{80} \mathrm{Kr},{ }^{82} \mathrm{Kr}$, ${ }^{83} \mathrm{Kr}$ and ${ }^{84} \mathrm{Kr}$ within the statistical uncertainty of $25 \mathrm{kHz}$. This uncertainty is quadratically added with the individual statistical errors giving rise to the following isotopes shifts: $301847(43) \mathrm{kHz}\left(f_{84 \mathrm{Kr}}-f_{80 \mathrm{Kr}}\right), 152403(35) \mathrm{kHz}$ $\left(f_{84 \mathrm{Kr}}-f_{82 \mathrm{Kr}}\right)$ and $98527(45) \mathrm{kHz}\left(f_{84 \mathrm{Kr}}-f_{83 \mathrm{Kr}}\right)$.

However, the ${ }^{86} \mathrm{Kr}$ isotope is strongly affected by the detector saturation effect due to the high abundance and therefore strong signal of the preceding ${ }^{84} \mathrm{Kr}$. Significant time was spend on characterizing this effect, and an attempt was made to model it. However, the modeling did not have enough predicting power to improve the accuracy. Therefore, we conservatively base our error on the variation seen between the different measurements at 
Table 1 Summary of the measurement result of the $4 p^{6} \rightarrow 4 p^{5} 5 p[1 / 2]_{0}$ two-photon transition in ${ }^{84} \mathrm{Kr}$. The values listed here are in $\mathrm{kHz}$, and the value in brackets denotes the $1 \sigma$ statistical uncertainty. The listed ${ }^{84} \mathrm{Kr}$ transition frequency is Doppler-free as explained in Sect. 5.3

\begin{tabular}{lll}
\hline${ }^{84} \mathrm{Kr}$ transition freq. & 2820833101688 \\
AC-Stark shift & -13 & $(58)$ \\
Laser phase shift & 1 & $(72)$ \\
Zeeman shift & 3 & $(35)$ \\
Detector saturation & 0 & $(13)$ \\
Total & 2820833101679 \\
\hline
\end{tabular}

different pulse delays and obtain an ${ }^{86} \mathrm{Kr}$ isotope shift of $f_{84 \mathrm{Kr}}-f_{86 \mathrm{Kr}}=-136.40(0.45) \mathrm{MHz}$.

The isotope shift uncertainties have been improved by a factor of 6.5, 4.2 and 3.7 for the isotopes ${ }^{80} \mathrm{Kr},{ }^{82} \mathrm{Kr}$ and ${ }^{83} \mathrm{Kr}$, respectively, compared to [17]. The present ${ }^{86} \mathrm{Kr}$ isotope shift determination is less precise than the previous measurement, due to the saturation effect discussed above.

\section{Conclusion}

Table 1 lists the different contributions to the atomicfrequency determination of ${ }^{84} \mathrm{Kr}$. The final result of the $4 p^{6} \rightarrow 4 p^{5} 5 p[1 / 2]_{0}$ transition in ${ }^{84} \mathrm{Kr}$, taking all corrections into account, is ${ }^{84} \mathrm{Kr}$ is $2,820,833,101,679(103) \mathrm{kHz}$. This corresponds to an improvement of the uncertainty by a factor of 34. It shows that high-accuracy spectroscopy with powerful short laser pulses down to deep-UV wavelengths is possible, and with a suppressed AC-Stark effect.

The accuracy of the present deep-UV spectroscopy demonstration is mainly limited by the short lifetime ( $27 \mathrm{~ns}$ ) of the excited state. If Ramsey-comb pulses with a longer time delay could be used (e.g., on longer lived states), then the accuracy improves accordingly. This feature gives good prospects for much more accurate spectroscopic measurements in, e.g., molecular hydrogen on the $\mathrm{EF} \leftarrow \mathrm{X}$ transition, where the longest lived states (with a low vibrational and rotational quantum number) have a lifetime of $\approx 200 \mathrm{~ns}$. Likewise, Ramsey-comb measurements could improve the determination of the fundamental vibrational splitting of $\mathrm{H}_{2}$ by at least an order of magnitude compared to previous measurements [37].

The demonstrated amplified pulse energy of $1.5 \mathrm{~mJ}$ per pulse is also enough for efficient HHG in gas jets, which makes RCS an interesting candidate to measure the $1 \mathrm{~S}-2 \mathrm{~S}$ transition in $\mathrm{He}^{+}$. The long lifetime of the $2 \mathrm{~S}$ excited state $(1.9 \mathrm{~ms})$ is very useful in this respect, as phase effects due to ionization in the HHG process can be avoided by choosing the pulses at least $200 \mathrm{~ns}$ apart. Each laser pulse then sees a fresh gas sample from the jet, and therefore a constant phase shift in the HHG process. Such a measurement could provide important new data to the proton radius puzzle, as the transition can then be compared to spectroscopy of muonic $\mathrm{He}^{+}$ions [13].

Acknowledgements The work in this paper was funded by the Foundation for Fundamental Research on Matter (FOM) through its Program 125: 'Broken Mirrors and Drifting Constants' and Projectruimte 12PR3098: 'Exploring the Boundaries of QED with Helium ${ }^{+}$.

Open Access This article is distributed under the terms of the Creative Commons Attribution 4.0 International License (http://creativecommons.org/licenses/by/4.0/), which permits unrestricted use, distribution, and reproduction in any medium, provided you give appropriate credit to the original author(s) and the source, provide a link to the Creative Commons license, and indicate if changes were made.

\section{References}

1. D. Hanneke, S. Fogwell, G. Gabrielse, New measurement of the electron magnetic moment and the fine structure constant. Phys. Rev. Lett. 100, 120801 (2008)

2. P.J. Mohr, B.N. Taylor, D.B. Newell, CODATA recommended values of the fundamental physical constants: 2010. Rev. Mod. Phys. 84(4), 1527-1605 (2012)

3. A. Wicht, J.M. Hensley, E. Sarajlic, S. Chu, A preliminary measurement of the fine structure constant based on atom interferometry. Phys. Scr. T102(1), 82-88 (2002)

4. R. Bouchendira, P. Cladé, S. Guellati-Khélifa, F. Nez, F. Biraben, New determination of the fine structure constant and test of the quantum electrodynamics. Phys. Rev. Lett. 106, 080801 (2011)

5. A. Matveev, C.G. Parthey, K. Predehl, J. Alnis, A. Beyer, R. Holzwarth, Th Udem, T. Wilken, N. Kolachevsky, Mi Abgrall, D. Rovera, C. Salomon, P. Laurent, G. Grosche, O. Terra, T. Legero, H. Schnatz, S. Weyers, B. Altschul, T.W. Hänsch, Precision measurement of the hydrogen $1 \mathrm{~S}-2 \mathrm{~S}$ frequency via a $920-\mathrm{km}$ fiber link. Phys. Rev. Lett. 110, 230801 (2013)

6. D.C. Yost, A. Matveev, A. Grinin, E. Peters, L. Maisenbacher, A. Beyer, R. Pohl, N. Kolachevsky, K. Khabarova, T.W. Hänsch, Th Udem, Spectroscopy of the hydrogen 1S-3S transition with chirped laser pulses. Phys. Rev. A 93, 042509 (2016)

7. S. Galtier, H. Fleurbaey, S. Thomas, L. Julien, F. Biraben, F. Nez, Progress in spectroscopy of the $1 \mathrm{~S}-3 \mathrm{~S}$ transition in hydrogen. J. Phys. Chem. Ref. Data 44(3), 031201 (2015)

8. D.Z. Kandula, C. Gohle, T.J. Pinkert, W. Ubachs, K.S.E. Eikema, Extreme Ultraviolet Frequency Comb Metrology. Phys. Rev. Lett. 105, 063001 (2010)

9. J. Biesheuvel, J-Ph Karr, L. Hilico, K.S.E. Eikema, W. Ubachs, J.C.J. Koelemeij, Probing QED and fundamental constants through laser spectroscopy of vibrational transitions in HD+. Nat. Commun. 7, 10385 (2016)

10. P. Beiersdorfer, Testing QED and atomic-nuclear interactions with high-Z ions. J. Phys. B At. Mol. Opt. 43(7), 074032 (2010)

11. R. Pohl, A. Antognini, F. Nez, F.D. Amaro, F. Biraben, J.M.R. Cardoso, D.S. Covita, A. Dax, S. Dhawan, L.M.P. Fernandes, A. Giesen, T. Graf, T.W. Hänsch, P. Indelicato, L. Julien, C. Kao, P. Knowles, E. Le Bigot, Y. Liu, J.A.M. Lopes, L. Ludhova, C.M.B. Monteiro, F. Mulhauser, T. Nebel, P. Rabinowitz, J.M.F. Dos Santos, L.A. Schaller, K. Schuhmann, C. Schwob, D. Taqqu, J.F.C.A. Veloso, F. Kottmann, The size of the proton. Nature 466, 213-216 (2010) 
12. A. Antognini, F. Nez, K. Schuhmann, F.D. Amaro, F. Biraben, J.M.R. Cardoso, D.S. Covita, A. Dax, S. Dhawan, M. Diepold, L.M.P. Fernandes, A. Giesen, A.L. Gouvea, T. Graf, T.W. Hänsch, P. Indelicato, L. Julien, C.-Y. Kao, P. Knowles, F. Kottmann, E.-O. Le Bigot, Y.-W. Liu, J.A.M. Lopes, L. Ludhova, C.M.B. Monteiro, F. Mulhauser, T. Nebel, P. Rabinowitz, J.M.F. dos Santos, L.A. Schaller, Ca Schwob, D. Taqqu, J.F.C.a Veloso, J. Vogelsang, R. Pohl, Proton structure from the measurement of $2 \mathrm{~S}-2 \mathrm{P}$ transition frequencies of muonic hydrogen. Science 339(6118), 417-420 (2013)

13. A. Antognini, K. Schuhmann, F.D. Amaro, P. Amaro, M. AbdouAhmed, F. Biraben, T.-L. Chen, D.S. Covita, A.J. Dax, M. Diepold, L.M.P. Fernandes, B. Franke, S. Galtier, A.L. Gouvea, J. Götzfried, T. Graf, T.W. Hänsch, M. Hildebrandt, P. Indelicato, L. Julien, K. Kirch, A. Knecht, F. Kottman, J.J. Krauth, Y.-W. Liu, J. Machado, C.M.B. Monteiro, F. Mulhauser, F. Nez, J.P. Santos, J.M.F. dos Santos, C.I. Szabo, D. Taqqu, J.F.C.A. Veloso, A. Voss, B. Weichelt, R. Pohl, Experiments towards resolving the proton charge radius puzzle. EPJ Web Conf. 113, 01006 (2016)

14. R. Pohl, F. Nez, L.M.P. Fernandes, D.A. Fernando, F. Biraben, J.M.R. Cardoso, D.S. Covita, A. Dax, S. Dhawan, M. Diepold, A. Giesen, A.L. Gouvea, T. Graf, T.W. Hänsch, P. Indelicato, L. Julien, P. Knowles, F. Kottmann, E.-O. Le Bigot, Y.-W. Liu, J.A.M. Lopes, L. Ludhova, C.M.B. Monteiro, F. Mulhauser, T. Nebel, P. Rabinowitz, J.M.F. dos Santos, L.A. Schaller, K. Schuhmann, C. Schwob, D. Taqqu, J.F.C.A. Veloso, A. Antognini, Laser spectroscopy of muonic deuterium. Science 353(6300), 669-672 (2016)

15. K. Pachucki, J. Komasa, Schrodinger equation solved for the hydrogen molecule with unprecedented accuracy. J. Chem. Phys. 144, 164306 (2016)

16. M. Herrmann, M. Haas, U.D. Jentschura, F. Kottmann, D. Leibfried, G. Saathoff, C. Gohle, A. Ozawa, V. Batteiger, S. Knünz, N. Kolachevsky, H.A. Schüssler, T.W. Hänsch, Th Udem, Feasibility of coherent XUV spectroscopy on the $1 \mathrm{~S}-2 \mathrm{~S}$ transition in singly ionized helium. Phys. Rev. A 79, 052505 (2009)

17. S. Witte, R.T. Zinkstok, W. Ubachs, W. Hogervorst, K.S.E. Eikema, Deep-ultraviolet quantum interference metrology with ultrashort laser pulses. Science 307, 400-403 (2005)

18. E. Peters, D.C. Yost, A. Matveev, T.W. Hänsch, Th Udem, Frequency-comb spectroscopy of the hydrogen 1S-3S and 1S-3D transitions. Ann. Phys. 525(7), L29-L34 (2013)

19. A. Cingoz, D.C. Yost, T.K. Allison, A. Ruehl, M.E. Fermann, I. Hartl, J. Ye, Direct frequency comb spectroscopy in the extreme ultraviolet. Nature 482, 68-71 (2012)

20. J. Lee, D.R. Carlson, R.J. Jones, Optimizing intracavity high harmonic generation for XUV fs frequency combs. Opt. Express 19(23), 23315 (2011)

21. A. Ozawa, W. Schneider, T.W. Hänsch, Th Udem, P. Hommelhoff, Phase-stable single-pass cryogenic amplifier for high repetition rate few-cycle laser pulses. N. J. Phys. 11, 083029 (2009)
22. A. Ruehl, A. Marcinkevicius, M.E. Fermann, I. Hartl, 80 W, $120 \mathrm{fs}$ Yb-fiber frequency comb. Opt. Lett. 35(18), 3015-3017 (2010)

23. J. Morgenweg, I. Barmes, K.S.E. Eikema, Ramsey-comb spectroscopy with intense ultrashort laser pulses. Nat. Phys. 10, 30-33 (2014)

24. R.K. Altmann, S. Galtier, L.S. Dreissen, K.S.E. Eikema, Highprecision Ramsey-comb spectroscopy at deep ultraviolet wavelengths. Phys. Rev. Lett. 117(17), 173201 (2016). doi:10.1103/ PhysRevLett.117.173201

25. J. Morgenweg, K.S.E. Eikema, Ramsey-comb spectroscopy: theory and signal analysis. Phys. Rev. A 89, 052510 (2014)

26. N.F. Ramsey, A molecular beam resonance method with separated oscillating fields. Phys. Rev. 78(6), 695-699 (1950)

27. S.A. Diddams, D.J. Jones, J. Ye, S.T. Cundiff, J.L. Hall, J.K. Ranka, R.S. Windeler, R. Holzwarth, Th Udem, T.W. Hänsch, Direct link between microwave and optical frequencies with a $300 \mathrm{THz}$ femtosecond laser comb. Phys. Rev. Lett. 84, 5102-5105 (2000)

28. R. Holzwarth, Th Udem, T.W. Hänsch, J.C. Knight, W.J. Wadsworth, P.S.J. Russell, Optical frequency synthesizer for precision spectroscopy. Phys. Rev. Lett. 85, 2264-2267 (2000)

29. R. Wynands, S. Weyers, Atomic fountain clocks. Metrologia 42, S64-S79 (2005)

30. S. Witte, RTh Zinkstok, A.L. Wolf, W. Hogervorst, W. Ubachs, K.S.E. Eikema, A source of 2 terawatt, 2.7 cycle laser pulses based on noncollinear optical parametric chirped pulse amplification. Opt. Express 14(18), 8168-8177 (2006)

31. J. Morgenweg, K.S.E. Eikema, Multi-delay, phase coherent pulse pair generation for precision Ramsey-frequency comb spectroscopy. Opt. Express 21(5), 5275-5286 (2013)

32. J. Morgenweg, K.S.E. Eikema, Tailored pulse sequences from an $880 \mathrm{~nm}$ pumped $\mathrm{Nd}: \mathrm{YVO}_{4}$ bounce amplifier. Opt. Lett. 37(2), 208-210 (2012)

33. J. Morgenweg, K.S.E. Eikema, A $1.8 \mathrm{~mJ}$, picosecond Nd:YVO4 bounce amplifier pump front-end system for high-accuracy XUV-frequency comb spectroscopy. Laser Phys. Lett. 5(11), 781-785 (2012)

34. I.N. Ross, P. Matousek, Geoffrey, H.C. New, K. Osvay, Analysis and optimization of optical parametric chirped pulse amplification. J. Opt. Soc. Am. B 19(12), 2945-2956 (2002)

35. A. Renault, D.Z. Kandula, S. Witte, A.L. Wolf, RTh Zinkstok, W. Hogervorst, K.S.E. Eikema, Phase stability of terawatt-class ultrabroadband parametric amplification. Opt. Lett. 32(16), 2363-2365 (2007)

36. S. Hannemann, E.J. Salumbides, W. Ubachs, Reducing the firstorder Doppler shift in a Sagnac interferometer. Opt. Lett. 32(11), 1381-1383 (2007)

37. G.D. Dickenson, M.L. Niu, E.J. Salumbides, J. Komasa, K.S.E. Eikema, K. Pachucki, W. Ubachs, Fundamental vibration of molecular hydrogen. Phys. Rev. Lett. 110, 193601 (2013) 